\title{
Total White Hypopyon: an Unusual Presentation of Phacolytic Glaucoma
}

\author{
Safinaz Mohd Khialdin, Aida Z Mohd Zahidin, Jemaima Che Hamzah, Ropilah Abdul Rahman \\ Ophthalmology Department, Pusat Perubatan Universiti Kebangsaan Malaysia, \\ Kuala Lumpur, Malaysia
}

\begin{abstract}
Phacolytic glaucoma normally presents with clinical features of eye pain and redness, anterior chamber inflammation, and increased intraocular pressure. This report is of a patient with phacolytic glaucoma, which presented as a total white hypopyon in a non-inflamed eye. Other possible causes of pseudohypopyon in this age group such as endogenous endophthalmitis or Masquerade syndrome were excluded before the patient underwent extracapsular cataract extraction with secondary posterior chamber intraocular lens implantation. She achieved satisfactory visual acuity postoperatively.
\end{abstract}

Key words: Cataract, Glaucoma, open-angle, Hypopyon

Asian J Ophthalmol. 2011;12:218-20.

\section{Introduction}

Phacolytic glaucoma is a form of secondary open angle glaucoma associated with hypermature cataract. Due to the presence of the white liquefied cortical matter, it can present as a pseudohypopyon that mimics other ocular diseases. A hypopyon could be inflammatory, infective, or neoplastic in aetiology. The underlying causes should be determined as the course, treatment, and prognosis are different.

\section{Case Report}

A 72-year-old woman presented with a sudden whitish discolouration of her left cornea 3 days previously. The vision in her left eye had been poor for the past 1 year. She had no history of fever or recent eye trauma. She had no significant appetite or weight loss. She had undergone cataract removal with intraocular lens (IOL) implantation in her right eye 10 years previously. Additionally, the patient had diabetes and was treated with oral hypoglycaemic agents.

Her best-corrected visual acuity (BCVA) was 6/18 in the right eye and light perception with good projection in the left eye. Both eyes were white. There was a total white hypopyon in the left eye (Figure 1). The cornea was clear and not oedematous. No keratic precipitates were seen. Her intraocular pressure (IOP) was

Correspondence: Dr Safinaz Mohd Khialdin, Ophthalmology Department, Pusat Perubatan Universiti Kebangsaan Malaysia, Jalan Yaakob Latif, Bandar Tun Razak, Cheras 56000, Kuala Lumpur, Malaysia. Tel: $(60$ 19) 2600313 ;

E-mail: drsafinaz_1978@yahoo.com.my
Figure 1. Total white hypopyon in a non-inflamed eye.

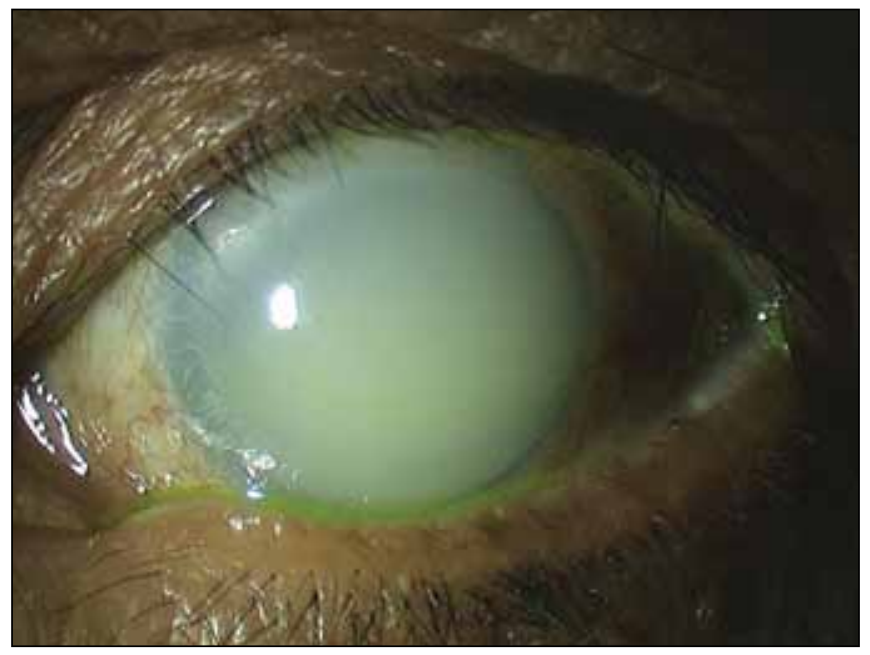

$28 \mathrm{~mm} \mathrm{Hg}$ in the right eye and $48 \mathrm{~mm} \mathrm{Hg}$ in the left eye. B-scan ultrasonography showed no evidence of vitreous opacity, retinal detachment, or choroidal mass in the left eye. The right eye was pseudophakic.

A clinical diagnosis of left phacolytic glaucoma was made. However, endogenous endophthalmitis and Masquerade syndrome still needed to be excluded. Systemic examination was unremarkable, and full blood count did not suggest any haematologic malignancy. Abdominal ultrasound did not show signs of liver abscess suggestive of endogenous endophthalmitis.

The patient was given maximum medical therapy (oral acetazolamide $250 \mathrm{mg} 4$ times daily, topical timolol maleate $0.5 \%$ twice daily, topical brimonidine tartrate $0.15 \%$ twice daily, and 
Figure 2. A clear anterior chamber 1 day postoperation.

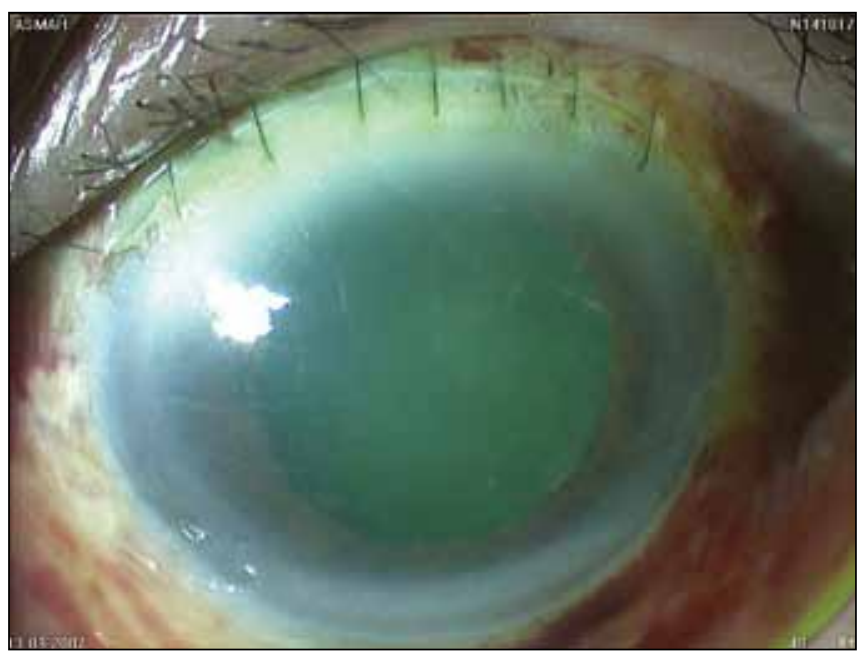

topical latanaprost $0.005 \%$ once daily) to lower the IOP, and also received topical dexamethasone $0.1 \%$ every 2 hours. She underwent anterior chamber washout with extracapsular cataract extraction (ECCE). Intraoperatively, there was liquefied cortical matter filling the entire anterior chamber. Morgagnian cataract with an intact capsule was visualised following the washout. No IOL was implanted pending the cytology and culture results of the anterior chamber fluid. Postoperatively, the anterior chamber was clear (Figure 2). Cytology results revealed no malignant cells, and culture was negative for microorganisms. Gonioscopic examination was performed and revealed intermittent peripheral anterior synechiae in the superior quadrant and grade 3 open angles in the other quadrants. Postoperatively, her IOP was $12 \mathrm{~mm} \mathrm{Hg}$ with the use of topical timolol maleate $0.5 \%$ and brimonidine tartrate $0.15 \%$ twice daily. She underwent secondary sulcus-fixated IOL implantation 4 months later. Her BCVA in the left eye improved to $6 / 12$.

\section{Discussion}

Phacolytic glaucoma is characterised by elevated IOP associated with a hypermature cataract, and is due to the leakage of lenticular material from hypermature or Morgagnian cataracts through an intact lens capsule. ${ }^{1}$ Typically, Morgagnian cataract consists of a capsular sac containing a free floating nucleus in the midst of a completely liquefied milky cortex. Nowadays, Morgagnian cataracts are less frequently encountered as cataract removal is done much earlier and fewer lenses progress to the hypermature stage.

The pathogenesis of phacolytic glaucoma is attributed to an obstruction of the inter-trabecular spaces by macrophages distended with engulfed lens material escaped from the lens. Epstein et al has identified high levels of high-molecular weight proteins that can directly obstruct the aqueous outflow channels in patients with mature or hypermature cataracts undergoing cataract surgery for presumed phacolytic glaucoma. ${ }^{2}$

The disease typically presents as a red and painful eye caused by acute inflammation and rise in IOP. ${ }^{3}$ However, in this patient, the eye was not inflamed, which might suggest a chronic and insidious onset. Sometimes, patients may also present with headache, nausea, and vomiting, similar to presentations of acute angle closure glaucoma. The gradual decline of visual acuity in the affected eye reflects the slow maturation process of the cataract. However, poor visual acuity at presentation is not an indicator for a poor prognosis. Eyes with poor light perception may obtain good visual acuity after removal of the cataract. ${ }^{4}$

In phacolytic glaucoma, there is usually a mature or hypermature cataract, corneal oedema secondary to elevated IOP, intense cells, and flare. The angles are usually open. Sometimes, there may be soft white patches of macrophage aggregates on the lens capsule. Clumps of white material may be noted floating in the anterior chamber, occasionally forming a hypopyon. In one retrospective series, 3 of 135 eyes with phacolytic glaucoma presented as hypopyon. ${ }^{5}$

This patient had an atypical presentation of a total white hypopyon in a non-inflamed eye. Hypopyon is defined as the inferior accumulation of pus resulting from suppurative infection in the anterior chamber. Hypopyon may also be caused by inflammatory mechanisms, and does not necessarily contain microorganisms. ${ }^{6}$ The term pseudohypopyon is used when the pus accumulation largely consists of neoplastic cells due to ocular tumour (Masquerade syndrome). True hypopyon and pseudohypopyon are encountered in various ocular or systemic diseases, including trauma, rheumatological disease, endogenous or exogenous infections, non-infective vasculitis, and neoplasm. ${ }^{7}$ Clinicians must be alert to life- and sightthreatening conditions, especially in patients who have underlying risk factors. Malignancy and infection were important differential diagnosis for this patient in view of her age and underlying diabetes mellitus.

Definitive management requires removal of the cataract. To avoid operating on an inflamed eye with high IOP, the initial management should concentrate on reducing the IOP and inflammation with antiglaucoma medication(s) and topical corticosteroids. ECCE with posterior chamber IOL implantation can lead to good results. ${ }^{8}$ The BCVA in this patient was 6/12 after the secondary IOL implantation. The IOP may revert to normal following removal of the cataract. However, the patient may develop glaucoma secondary to peripheral anterior synechiae formation or ischaemic anterior chamber angles. ${ }^{9}$

In conclusion, phacolytic glaucoma can present as a pseudohypopyon in a non-inflamed eye, and other serious conditions such as endogenous endophthalmitis and malignancy must be excluded. 
A rational clinical approach to diagnosis is important so that appropriate treatment can be instituted.

\section{References}

1. Ellant JP, Obstbaum SA. Lens-induced glaucoma. Doc Ophthalmol. 1992;8:317-38.

2. Epstein DL, Jedziniak JA, Grant WM. Identification of heavymolecular weight soluble protein in aqueous humor in human phacolytic glaucoma. Invest Ophthalmol Vis Sci. 1978;17:398-402.

3. Flocks M, Littwin CS, Zimmerman LE. Phacolytic glaucoma: a clinicopathological study of 138 cases of glaucoma associated with hypermature cataract. Arch Ophthalmol. 1955;54:37-45.

4. Papaconstantinou D, Georgalas I, Kourtis N, et al. Lens-induced glaucoma in the elderly. Clin Interv Aging. 2009;4:331-6.

5. Braganza A, Thomas R, George T, Mermoud A. Management of phacolytic glaucoma: experience of 135 cases. Indian J Ophthalmol. 1998;46:139-43.

6. Agrawal RV, Murthy S, Sangwan V. Current approach in diagnosis and management of anterior uveitis. Indian J Ophthalmol. 2010;58: 11-9.

7. Ramsay A, Lightman S. Hypopyon uveitis major review. Surv Ophthalmol. 2001;46:1- 18.

8. Singh G, Kaur J, Mall S. Phacolytic glaucoma - its treatment by planned extracapsular cataract extraction with posterior chamber intraocular lens implantation. Indian J Ophthalmol. 1994;42:145-47.

9. Krishnadas R, Ramakrishnan R. Secondary glaucomas: the tasks ahead. Community Eye Health. 2001;14:40-2. 